Rev. Elev. Méd. vét. Pays trop., 1979, 32 (4) : 371-378.

\title{
Anoplocéphalides parasites de l'intestin et des canaux biliaires des herbivores sauvages d'Afrique centrale
}

\author{
par M. GRABER et J. THAL
}

\begin{abstract}
RÉSUMÉ
La présente étude passe en revue les Cestodes de la famille des Anoplocephalidae recueillis entre 1954 et $1972 \mathrm{chez}$ les antilopes et chez les buffles (au total 314) d'Afrique centrale (République Centrafricaine, Tchad, Nord Cameroun).

Onze espèces différentes ont été inventoriées.

Stilesia hepatica, l'agent de la stilesiose hépatique, affecte un animal sur huit. On le trouve principalement chez l'hippotrague $(85 \mathrm{p} .100)$ et le waterbuck (68 p. 100), plus rarement chez le redunca et le cob de Buffon (10 p. 100).

Le téniasis intestinal est à base de Stilesia globipunctata, d'Avitellina (surtout centripunctata), de Moniezia et de Thysaniezia ovilla. Il frappe un ruminant sut cinq. La plupart des espèces sont atteintes dans des proportions variables avec, parfois, des taux élevés (de 40 à 70 p. 100) notamment chez la gazelle darna, l'ourébi, le céphalophe couronné, l'oryx et l'hippotrague.

Les auteurs donnent quelques renseignements sur la répartition géographique de ces Cestodes, ainsi que sur leur rôle pathogène qui, sauf exception, parait peu important.

lls comparent le téniasis des ruminants domestiques et le téniasis des ruminants sauvages de cette région d'Afrique.
\end{abstract}

\section{INTRODUCTION}

La collection de parasites d'herbivores sauvages rassemblée, de 1969 à 1972, dans le centre et le Sud du Tchad (Drs Provost, Borredon et Chailloux) au Nord Cameroun (Dr Macon) et dans l'Est de la République Centrafricaine (R. C. A.) (19) comportait, entre autres, un certain nombre de cestodes appartenant à la famille des Anoplocephalidae.

Le téniasis qu'ils provoquent est, en Afrique tropicale, une affection banale des ruminants domestiques (3). Chez les ruminants sauvages, elle est beaucoup moins bien connue. Aussi, les renseignements fournis par cette enquête et les observations faites précédemment de 1954 à $1969(4,5,8)$ permettent-ils, dans une certaine mesure, de combler cette lacune.

Service de Parasitologie, Ecole Nationale Vétérinaire de Lyon, Marcy L'Etoile, 69260 Charbonnières-les-Bains.

I. E. M. V. T., 10, rue Plerre-Curie, 94700 MaisonsAlfort, France.

\section{MATÉRIEL ET MÉTHODE}

\section{Matériel}

Cent cinquante-sept autopsies complètes ont été effectuées se répartissant ainsi :

Bubalus (Syncerus) caffer, Sparrman, le buffle : 81 (R. C. A., 71 ; Tchad, 9 ; Nord Cameroun, 1).

Alcelaphus lelwel, Heuglin, le bubale: 15 (R. C. A., 13 ; Tchad, 1 ; Nord Cameroun, 1).

Kobus defassa, Rüppel, le waterbuck ou cob defassa : 11 (R. C. A., 7 ; Tchad, 4).

Hippotragus equinus, Desmarest, l'hippotrague: 11 (R. C. A., 7; Tchad, 3 ; Nord Cameroun, 1).

Kobus cob, Erxleben, le cob de Buffon : 10 (R. C. A., 5 ; Tchad, 5).

Ourebia ourebi, Zimmerman, l'ourébi : 8 (R. C. A., 3 ; Tchad, 1 ; Nord Cameroun, 4). 
Redunca redunca nigeriensis, Blaine: le redunca : 5 (R. C. A., 2 ; Tchad, 3).

Sylvicapra grimmia, Linné, le céphalophe couronné : 5 (R. C. A., 3 ; Nord Cameroun, 2).

Tragelaphus scriptus, Pallas, le guib: 4 (R. C. A., 3 ; Nord Cameroun, 1).

Taurotragus derbianus, Gray, l'éland de Derby : 4 (R. C. A., 3 ; Nord Cameroun, 1).

Damaliscus korrigum, Ogilby, le damalisque : 2 (R. C. A., 1 ; Tchad, 1).

Gazella rufifrons, Gray, la gazelle corinne : 1 (Tchad).

\section{Méthode}

Les cestodes ont été déterminés après coloration au carmin chlorhydrique de plusieurs fragments prélevés en différents points de la chaîne et montage au baume du Canada.

\section{LES PARASITES}

Les Anoplocephalidés rencontrés se localisent dans l'intestin et dans les canaux biliaires.

\section{Cestodes de l'intestin}

1.1. Moniezia expansa Rudolphi, 1810 (Anoplocephalidae : Anoplocephalinae)

Origine du matériel étudié : R. C. A.

- Un exemplaire récolté chez un Syncerus caffer (Coord. (*) $236 \mathrm{C} \mathrm{d}$ ).

La présence de nombreuses glandes interproglotidiennes en rosette permet d'identifier facilement le parasite.

1.2. Moniezia benedeni Moniez, 1879 (Anoplocephalidae: Anoplocephalinae)

Origine du matériel étudié : Nord Cameroun.

- Trois exemplaires récoltés chez Taurotragus derbianus (Coord. $148 \mathrm{D} \mathrm{a}$ ).

\subsection{Avitellina centripunctata Rivolta, 1874 (Anoplocephalidae: Thysanosominae)}

Origine du matériel étudié :

a) R.C.A.

- Nombreux exemplaires récoltés chez 10 Syncerus caffer dans toute la zone cynégé-

(*) Coordongées préconisées par l'I. B. A. H. qui utilise des carrés délimités par les longitudes et par les latitudes. tique, du Bamingui (Coord. $207 \mathrm{C} \mathrm{b}$ ) à la rivière Chinko (Coord. $245 \mathrm{C} \mathrm{b}$ ), chez deux Alcelaphus lelwel (Coord. $218 \mathrm{D}$ a et $238 \mathrm{~A} \mathrm{~d}$ ), chez un Kobus cob (Coord. 219 B b) et chez un Sylvicapra grimmia (Coord. $218 \mathrm{D} \mathrm{c}$ ).

b) Tchad

- Une dizaine d'exemplaires récoltés chez deux Syncerus caffer (Coord. $199 \mathrm{~A} \mathrm{~b}$ ).

\section{c) Nord Cameroun}

- Une vingtaine d'exemplaires récoltés chez un Hippotragus equinus (Coord. $139 \mathrm{~A}$ a).

Sous le binôme Avitellina centripunctata, SPASSKI (18) regroupe un certain nombre d'espèces décrites précédemment en Asie et en Afrique (2), notamment Avitellina sudanea et Avitellina woodlandi. Il s'agit donc d'une espèce polymorphe comme le montre l'étude du matériel recueilli en Afrique centrale.

Tous ces cestodes possèdent dans la région antérieure du corps, en arrière du scolex, deux paires de canaux excréteurs longitudinaux, à savoir deux canaux dorsaux placés à l'intérieur de deux canaux ventraux beaucoup plus larges. Les premiers disparaissent peu à peu et il ne reste plus, dans la région postérieure du strobile, que les deux canaux ventraux dont le diamètre peut atteindre 200-300 $\mu$.

Dans les segments mûrs, les testicules sont groupés en rangées transversales, de part et d'autre des vaisseaux excréteurs ventraux. Leur superposition donne naissance à quatre colonnes longitudinales de testicules disposées à l'intérieur des canaux excréteurs (colonnes internes) ou à l'extérieur (colonnes externes).

La poche du cirre d'Avitellina centripunctata " sensu stricto » est plus courte (100-120 $\mu$ ) que la vulve. Les colonnes externes comprennent deux-trois testicules par rangée et les colonnes internes quatre-sept. Ces caractères sont ceux des Avitellina d'antilopes (Bubale, hippotrague, céphalophe couronné) en R. C. A. et au Nord Cameroun, plus rarement des Avitellina de buffles en R. C. A. (un quart des cas).

La « forme sudanea » ne diffère de la précédente que par ses organes parutérins réniformes et par ses colonnes externes ne renfermant qu'un testicule par rangée. La moitié des buffles de R. C. A., la totalité des buffles du Tchad sont porteurs d'Avitellina centripunctata pouvant être rapportés à cette forme.

Quant à la «forme woodlandi", elle affecte le quart des buffles de R. C. A. et, au Tchad, 


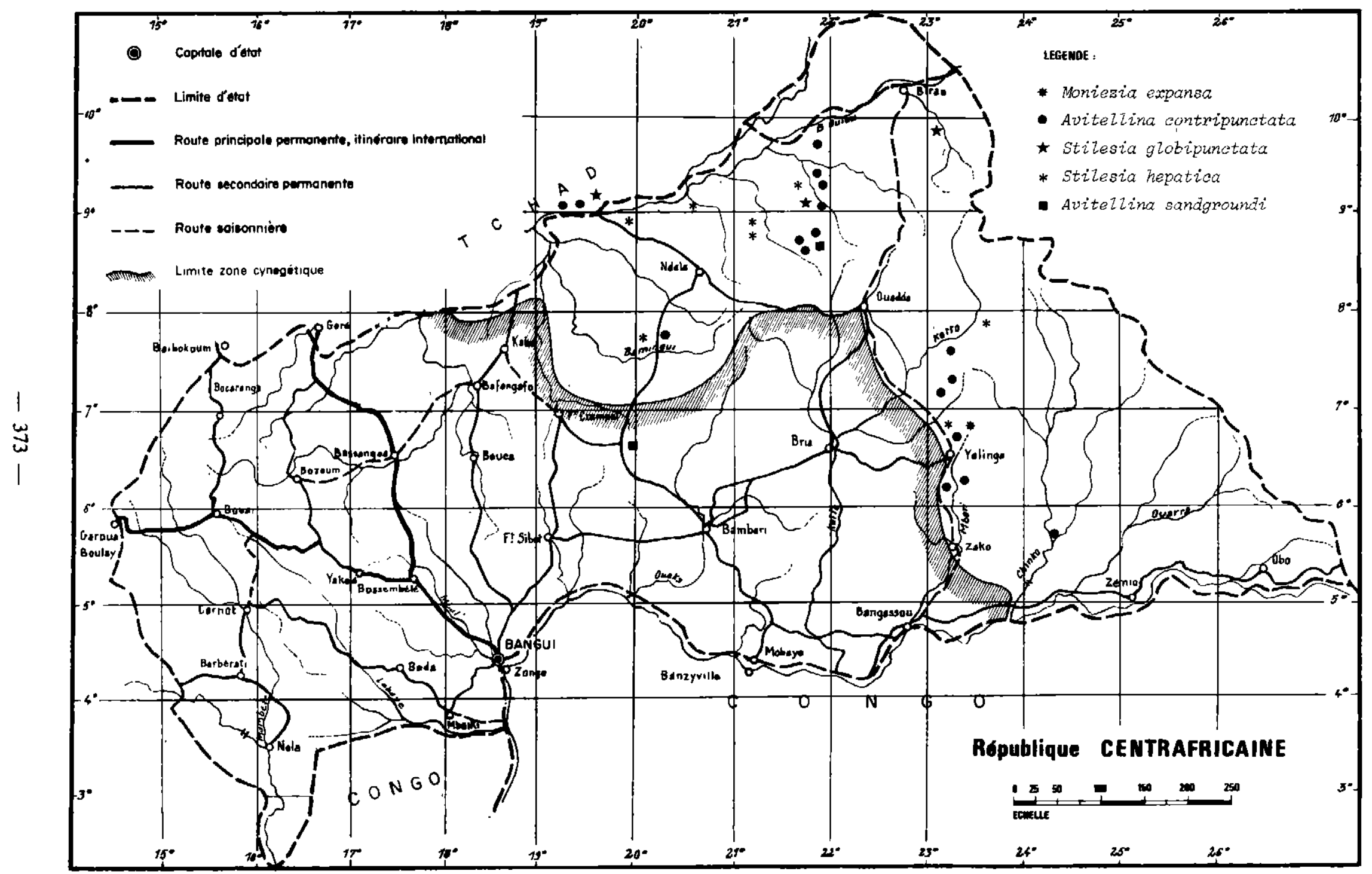


l'addax, l'oryx et la gazelle dama $(6,7)$. La poche du cirre est de deux à trois fois plus longue (180-300 $\mu$ ) que la vulve et les colonnes externes sont formées de trois à quatre testicules par rangée.

1.4. Avitellina sandgroundi Woodland, 1935 (Anoplocephalidae : Thysanosominae)

Origine du matériel étudié : R. C. A.

- Six exemplaires, mûrs et immatures, récoltés chez trois Hippotragus equinus (Coord. $196 \mathrm{D} \mathrm{b} ; 219 \mathrm{D} \mathrm{b} ; 218 \mathrm{D} \mathrm{b})$.

Avitellina sandgroundi se différencie d'Avitellina centripunctata par l'existence d'une seule paire de canaux excréteurs, les canaux ventraux, par un scolex massif pourvu de quatre ventouses de $900 \mu$ de diamètre, par le nombre de testicules dans chaque colonne (colonnes externes, une-deux par rangée ; colonnes internes, troiscinq) et par l'extrême brièveté des segments constituant le strobile (21).

\subsection{Avitellina edifontaineus Woodland, 1928 (Anoplocephalidae : Thysanosominae)}

Origine du matériel étudié : Nord Cameroun.

— une vingtaine d'exemplaires récoltés chez Taurotragus derbianus (Coord. $148 \mathrm{D}$ a). La description originale de WOODLAND (20) est incomplète, car l'auteur, faute de matériel, n'a pu examiner ni le scolex, ni le cou du parasite.

Dans le lot d'Avitellina recueillis chez l'éland de Derby, plusieurs scolex ont pu être isolés. Montés dans le baume, ils mesurent $600 \mu$ en moyenne et les ventouses, presque rondes, $240-288 \times 216-264 \mu$ (moyenne, $264 \times 250 \mu$ ). Immédiatement en arrière du scolex, on note la présence de deux paires de canaux excréteurs. Les canaux dorsaux sont minces $(3 \mu)$, presque invisibles et disparaissent à $0,5-0,8 \mathrm{~mm}$ du scolex. La largeur des canaux ventraux qui varie de 10 à $15 \mu$ dans la région céphalique augmente peu à peu pour atteindre, dans les segments gravides, 210-250 $\mu$.

Les autres caractères sont ceux donnés par WOODLAND (20) : longueur du cestode, plus d'un mètre ; largeur, $3,3 \mathrm{~mm}$. Les colonnes externes de testicules (un seul par rangée) sont discontinues et irrégulières avec des «trous» de $0,5-1 \mathrm{~mm}$. Les colonnes internes comprennent par rangée, neuf testicules de $80 \mu$. La poche du cirre, dorsale à la vulve $(120 \mu)$, est longue (240.250 $\mu$ ).
1.6. Stilesia globipuncata Rivolta, 1874 (Anoplocephalidae: rhysanosominae)

Origine du matériel étudié :

a) R. C.A.

- Plusieurs centaines d'exemplaires récoltés chez deux Ourebia ourebi (Coord. $219 \mathrm{~B}$ b; $229 \mathrm{C} \mathrm{c}$ ).

b) Tchad

- Quelques dizaines d'exemplaires récoltés chez un Ourebia ourebi (Coord. 199 A b) et chez un Kobus cob (Coord. 1710 A c).

\section{c) Nord Cameroun}

- Plusieurs centaines d'exemplaires récoltés chez quatre Ourebia ourebi (Coord. 139 A a et $148 \mathrm{D}$ a).

\section{Cestodes des canaux biliaires}

Stilesia hepatica Wollfhügel, 1903 (Anoplocephalidae: Anoplocephalinae)

Origine du matériel étudié :

a) R.C.A.

- Soixante-quinze exemplaires récoltés chez cinq Hippotragus equinus (Coord. $198 \mathrm{D} \mathrm{d}$, $198 \mathrm{D} \mathrm{b}, 218 \mathrm{C} \mathrm{d}, 219 \mathrm{~B} \mathrm{~b}, 239 \mathrm{C} \mathrm{c}$ ) ; deux exemplaires récoltés chez un Kobus cob (Coord. $207 \mathrm{C} \mathrm{b}$ ) et cent vingt chez sept Kobus defassa (Coord. $209 \mathrm{~B} \mathrm{a}, 219 \mathrm{~B} \mathrm{~b}, 237 \mathrm{D}$ c, $236 \mathrm{C} \mathrm{c}$ et $236 \mathrm{C} \mathrm{d}$ ).

\section{b) Tchad}

- Quarante-six exemplaires récoltés chez trois Hippotragus equinus (Coord. $199 \mathrm{~B}$ a et $1710 \mathrm{~A} \mathrm{c}$ ) et trente-cinq chez deux Kobus defassa (Coord. $199 \mathrm{~B}$ a).

\section{c) Nord Cameroun}

- Un exemplaire récolté chez un Hippotragus equinus (Coord. $139 \mathrm{~A}$ a).

\section{COMMENTAIRES}

1. Depuis 1954, les enquêtes menées en Afrique centrale (Tchad, Nord Cameroun, R. C. A.) sur environ trois cent quatorze herbivores sauvages $(4,5,8,19)$ ont permis de mettre en évidence les cestodes suivants : 


\subsection{Dans l'intestin}

- Moniezia monardi chez l'hippotrague et le cob defassa.

- Moniezia expansa chez le buffle et l'Oryx.

- Moniezia benedeni chez l'éland de Derby.

- Avitellina edifontaineus chez l'éland de Derby.

- Avitellina centripunctata chez le buffle, le bubale, l'hippotrague, le cob de Buffon, le céphalophe couronné, la gazelle dama, l'addax et l'oryx.

- Avitellina sandgroundi chez l'hippotrague, le bubale et le damalisque.

- Avitellina buechneri chez le cob defassa et le cob de Buffon.

- Stilesia globipunctata chez le waterbuck, l'hippotrague, le cob de Buffon, l'ourébi, le céphalophe couronné, la gazelle corinne et la gazelle dama.

- Thysaniezia ovilla chez la gazelle dama.

\subsection{Dans les canaux biliaires}

- Crossotaenia baeri chez le guib.

- Stilesia hepatica chez le waterbuck, l'hippotrague, le cob de Buffon et le redunca.

En Afrique au sud du Sahara, ces Anoplocephalidés ont déjà été signalés à de multiples reprises et une liste aussi exhaustive que possible a été dressée par ROUND (14). A cette inventaire, il faut ajouter plusieurs Cestodes découverts, ces 10 dernières années, chez des hôtes nouveaux :

- Moniezia expansa chez Redunca fulvorufula en Afrique du sud (1) et chez Nesotragus moschatus, le Suni au Kenya (11).

Moniezia benedeni chez Tragelaphus strepsiceros, le koudou, en Zambie et au Zimbabwé (14), ainsi que chez le guib au Mozambique (17).

- Avitellina centripunctata chez Damaliscus albifrons en Afrique du sud (9), chez Gazella soemmeringi, la gazelle de Soemmering, en Ethiopie (6) et chez le guib au Mozambique (17).

- Avitellina sandgroundi chez Hippotragus niger au Zimbabwé et en Zambie (13).

- Stilesia globipunctata chez la gazelle de Soemmering en Ethiopie (6).

- Stilesia hepatica chez l'éland du Cap et Connochaetes taurinus, le gnou en Tanzanie (10, 16).
En Afrique centrale, doivent être considérés comme des hôtes nouveaux :

- Pour Moniezia expansa, le buffle.

- Pour Moniezia benedeni et Avitellina edifontaineus, l'éland de Derby.

- Pour Moniezia monardi, l'hippotrague et le waterbuck.

- Pour Avitellina centripunctata, la gazelle dama, l'addax et le cob de Buffon.

-- Pour Avitellina sandgroundi, le bubale et le damalisque.

- Pour Avitellina buechneri, le waterbuck.

- Pour Stilesiaglobipunctata, le cob de Buffon, le waterbuck, la gazelle corinne, la gazelle dama et le céphalophe couronné.

- Pour Thysaniezia ovilla, la gazelle dama.

- Pour Crossotaenia baeri, le guib.

C'est la première fois que Moniezia monardi, Avitellina sandgroundi, Avitellina edifontaineus, Avitellina buechneri et Crossotaenia baeri sont signalés au nord de l'Equateur.

Il est bon de rappeler qu'en général, les Anoplocephalidés n'ont pas de spécificité d'hôte bien marquée. Cependant, en Afrique tropicale, Avitellina sandgroundi semble plus particulièrement inféodé à l'hippotrague, Avitellina edifontaineus à l'éland, Stilesia globipunctata aux petites antilopes et aux gazelles, Stilesia hepatica à l'hippotrague et aux cobs.

2. En Afrique centrale, la répartition géographique de ces Anoplocephalidés n'est pas uniforme. Ainsi, il est possible de rencontrer :

- Des Avitellina centripunctata du $5^{\mathrm{e}}$ au $14^{e}$ parallèle.

- Des Stilesia globipunctata du $9^{e}$ au $14^{e}$ parallèle.

- Des Sillesia hepatica du $6^{\mathrm{e}}$ au $12^{\mathrm{e}}$ parallèle, les zones sèches du Nord, du Centre et de l'Est du Tchad en étant presque totalement dépourvues. Cette remarque vaut également pour les ruminants domestiques, notamment les moutons, vivant dans ces régions. Le même phénomène a été observé au Kenya (16), pays où la stilésiose hépatique des ovins est beaucoupplus fréquente dans les zones à fortes précipitations que dans les zones plus sèches.

- Les autres Avitellina sont beaucoup plus localisés : entre le $8^{\mathrm{e}}$ et le $10^{\mathrm{E}}$ parallèle pour 
Avitellina sandgroundi et, entre le $9^{\mathrm{e}}$ et le $10^{\mathrm{e}} \mathrm{pa-}$ rallèle, pour Avitellina buechneri.

3. Les taux d'infestation varient sensiblement selon les espèces parasites et selon les hôtes.

3.1. Sur trois cent-quatorze ruminants sauvages (cent vingt-six en R. C. A. et cent quatrevingt huit au Tchad), la stilésiose hépatique sévit au taux moyen de 12 p. 100 , tant en R. C. A. qu'au Tchad. Ce taux est nettement supérieur à celui des animaux domestiques de la région : au Tchad (3), 0,06 p. 100 chez les bovins, 2,4 p. 100 chez les ovins, 0,5 p. 100 chez les chèvres, ce qui laisse supposer que, dans ces zones tout au moins (*), la stilésiose hépatique est plutôt le fait des ruminants sauvages que des ruminants domestiques: ceux-ci s'infestent là où les antilopes sont abondantes, de préférence au voisinage des cours d'eau et des lacs, c'està-dire dans les régions humides.

Le téniasis intestinal des herbivores sauvages qui touche, en moyenne, un animal sur cinq, est un peu plus conséquent au Tchad $(21,2$ p. 100) qu'en R. C. A. (16,6 p. 100). La nature du téniasis n'est pas la même : en R. C. A., les Stilesia et les Moniezia sont faiblement représentés (moins de 1,5 p. 100) au profit des Avitellina (15 p. 100). Au Tchad et au Nord Cameroun, les Avitellina et les Stilesia sont à égalité ( 9 p. 100), suivis des Moniezia et des Thysaniezia (de 1 à 2,5 p. 100).

L'importance et la structure du téniasis intestinal des ruminants domestiques sont totalement différentes. Dans l'Ouest de la R. C. A., chez le zébu, le taux moyen d'infestation est de 3 p. 100 et les cestodes en cause sont des Moniezia et des Thysaniezia (7). Au Tchad, et au Nord Cameroun (3), le pourcentage de zébus infestés (jeunes et adultes) est de 15,3 p. 100, avec également prédominance des Thysaniezia et des Moniezia, ce qui est aussi le cas des chèvres. Chez le mouton (67 p. 100 d'animaux parasités), les espèces les plus fréquemment rencontrées sont Avitellina centripunctata (43 p. 100), Stilesia globipunctata $(35,4$ p. 100$)$ et Moniezia sp. $(16,2$ p. 100).

3.2. Les diverses espèces de ruminants autopsiés au cours de ces enquêtes ne sont pas

(*) Au Kenya, 50 p. 100 des moutons sont porteurs de Stilesia hepatica (17). toutes infestées et aucun cestode n'a été recueilli chez la gazelle dorcas (nombre d'animaux autopsiés : 36) et chez le grand koudou (2).

3.2.1. Le téniasis intestinal touche faiblement (moins de 10 p. 100 ) le bubale $(0,9$ p. 100$)$; la gazelle rufifrons $(9,5$ p. 100$)$ et le damalisque I1 est un peu plus élevé (de 10 à 20 p. 100) chez le buffle du Tchad et de la R. C. A. (16 p. 100), le cob defassa du Tchad (20 p. 100) et le cob de Buffon du Tchad et de la R. C. A. (15 p. 100).

Les herbivores les plus atteints sont, dans l'ordre décroissant, la gazelle dama (75 p. 100) au Tchad, l'ourébi (61 p. 100) au Tchad, en R. C. A. et au Nord Cameroun, L'oryx (44 p. 100) au Tchad, le céphalophe couronné (42 p. 100) au Tchad et en R. C. A. et l'hippotrague ( 40 p. 100) au Tchad, en R. C. A. et au Nord Cameroun.

Ailleurs, en Afrique, on ne possède que peu de renseignements. Depuis le début du siècle, de nombreuses récoltes d'Anaplocephalidés ont été effectuées çà et là (14). Malheureusement, elles sont rarement accompagnées d'enquêtes statistiques, sauf en Afrique du Sud, au Parc National Kruger où 7 p. 100 des buffles sont porteurs d'Avitellina centripunctata et au Zimbabwé-Zambie (13) où les animaux hébergeant le plus grand nombre de cestodes intestinaux sont le céphalophe couronné, l'hippotrague, le steenbok et l'éland du Cap, le taux maximal d'infestation ne dépasse pas 30 p. 100.

3.2.2. Le téniasis à Stilesia hepatica affecte surtout l'hippotrague ( 85 p. 100) et le cob defassa (68 p. 100) au Tchad et en R. C. A. Il est peu répandu (10 p. 100) chez le cob de Buffon (R. C. A.) et chez le redunca (Tchad). Les autres espèces en sont indemnes.

4. Le niveau de l'infestation diffère considérablement d'une espèce à l'autre et d'un animal à l'autre. Les critères d'appréciation sont ceux définis par ROTH et DALCHOW (13),c'est-àdire :

- + (de un à vingt parasites) : infestation faible.

-++ (de vingt à cent parasites) : infestation moyenne.

-+++ (plus de cent parasites) : infestation massive.

Les observations faites en Afrique centrale sont résumées dans le tableau suivant : 


\begin{tabular}{|c|c|c|c|}
\hline Parasites & \multicolumn{3}{|c|}{ Hôtes et niveau de 1'infestation (p.100) } \\
\hline & Faible & Moyen & Massif \\
\hline $\begin{array}{l}\text { Moniezia } \\
\text { Avitelizna }\end{array}$ & $\begin{array}{c}84 \\
\text { (toutes espèces) }\end{array}$ & $\begin{array}{c}13 \\
\text { (Hippotrague, Buffle } \\
\text { Elan de Derby) }\end{array}$ & $\begin{array}{c}3 \\
\text { (Buffle) }\end{array}$ \\
\hline $\begin{array}{l}\text { Stilesia } \\
\text { mlobipunctata }\end{array}$ & $\begin{array}{c}62,5 \\
\text { (toutes espèces) }\end{array}$ & (Qurëbi et GazeIles) & $\begin{array}{c}12,5 \\
\text { (Ourébi) }\end{array}$ \\
\hline Stilesii hepatioa & $\begin{array}{c}78,7 \\
\text { (toutes espẽces) }\end{array}$ & $\begin{array}{r}19,3 \\
\text { (Hippotrague, } \\
\text { Waterbuck) }\end{array}$ & $\stackrel{2}{2}$ \\
\hline Total & 77,6 & 17,6 & 4,8 \\
\hline
\end{tabular}

Les Avitellina et les Moniezia, en raison de leur petit nombre, n'ont, sauf exception, qu'un rốle pathogène limité. La stilésiose duodénale pourrait être plus grave, surtout chez l'ourébi et chez les gazelles. Des observations faites chez le mouton montrent que les scolex de Stilesia globipunctata s'enfoncent dans la muqueuse des premières portions de l'intestin en provoquant une inflammation catarrhale, l'épaississement de la paroi et la formation d'un nodule réactionnel d'où pend, dans la lumière de l'organe, l'extrémité postérieure des parasites. Lorsque les Stilesia sont nombreux, on note de l'anorexie, de la diarrhée, une cachexie profonde et, au dernier stade, la parésie du train postérieur avec démarche titubante.

Quant aux Stilesia hepatica, leur rôle pathogène est encore controversé. Le cestode occasionne l'hyperplasie des canaux biliaires avec formation de nodules sur leur trajet. En général le parenchyme hépatique demeure à peu près intact (12).

\section{CONCLUSIONS}

Plusieurs enquêtes effectuées de 1954 à 1972 en Afrique centrale (République Centrafricaine, Tchad, Nord Cameroun) ont permis de mettre en évidence chez les herbivores sauvages onze espèces différentes d'Anoplocephalidés parasites des canaux biliaires (deux espèces) et de l'intestin (neuf espèces). Avitellina edifontaineus est brièvement redécrit.

Stilesia hepatica, l'agent de la stilésiose hépatique, affecte un animal sur huit. Il se rencontre principalement chez l'hippotrague ( 85 p. 100) et le waterbuck ( 68 p. 100 ), plus rarement chez le redunca et le cob de Buffon (10 p. 100).

Le téniasis intestinal frappe un ruminant sur cinq. Interviennent, dans l'ordre décroissant, quatre espèces d'Avitellina, Avitellina centripunctata étant l'espèce dominante, Stilesia globipunctata, trois espèces de Moniezia et Thysaniezia ovilla.

Les herbivores sauvages sont atteints dans des proportions qui varient sensiblement selon les espèces. Les taux d'infestation oscillent, en général, entre 1 et 20 p. 100 . Parfois, ils sont plus élevés (de 40 à 75 p. 100), notamment chez la gazelle dama, l'ourébi, le céphalophe couronné l'oryx et l'hippotrague.

Dans les trois quarts des cas, le téniasis hépatico-intestinal des ruminants sauvages est de faible importance et semble assez bien supporté, ce qui n'est pas toujours le cas chez les ruminants domestiques, en particulier chez les moutons porteurs de Stilesia globipunctata.

\section{SUMMARY}

Tapeworms from wild ruminants in Central Africa

This work studies the species of Anoplocephalidae tapeworms collected between 1954 and 1972 in Central Africa (Central african Republic, Chad, North Cameroon) from antelopes and African buffaloes (three hundred and fourteen autopsies). Eleven species are inventoried.

Stilesio hepatica, the liver tapeworm, was found in 12 p. 100 of antelopes, principally in roan antelope $(85$ p. 100$)$ and waterbuck $(68$ p. 100), less frequently in reedbuck and cob (10 p. 100).

Intestinal teniasis which occurs in 20 p. 100 of game animals is caused by four species of Avitellina (mainly centripunctata), Stilesia globipunctata, three species of Moniezia and Thysaniezia ovilla. Almost all species of wild rumi- 
nants are affected, particularly grey duiker, oribi, dama gazelle, roan antelope and oryx (from 40 to 75 p. 100).

The authors give some informations on the geographic distribution and the pathogenic action of these tapeworms which is mild in most cases (more than 75 p. 100). Teniasis of wild and domestic ruminants is compared.

\section{RESUMEN}

\section{Anoplocephalidae, parásitos del intestino y de los canales biliares de los herbivoros salvajes de Africa central}

Este estudio pasa en revista los céstodos de la familia de los Anoplocephalidae recogidos entre 1954 y 1972 en los antílopes y en los búfalos (en resumen 314) de Africa central (República Centroafricana, Chad, norte de Camerún).

Se inventariaron once especies diferentes.

Stilesia hepatica, agente de la estilesiosis hepática, ataca un anımal de ocho; se le encuentra principalmente en Hippotragus equinus (85 p. 100) y en Kobus defassa (68 p. 100), más raramente en Redunca redunca y en Kobus cob (10 p. 100) Stilesia globipunctata, Avitellina (sobre todo centripunctata), Moniezia y Thysaniezia ovilla son causa principalmente del teniasis intestinal que ataca un rumiante de cinco.

Infestan la mayor parte de las especies a veces con proporciones elevadas (de 40 a 70 p. 100) especialmente en la gacela dama, Ourebia ourebi, Sylvicapra grimmia, Oryx e Hippotragus equinus.

Los autores dan algunos datos sobre la repartición geográfica de estos céstodos asi como sobre su papel patógeno que, salvo excepción, parece poco importante.

Comparan el teniasis de los rumiantes domésticos con el de los rumiantes salvajes de esta región de Africa.

\section{BIBLIOGRAPHIE}

1. BAKER (M. K.), BOOMKER (J.). Helminths from the mountain reedbuck, Redunca fulvorufula Afzelius 1815. Onderstepoort J. vet. Res., 1973, 40 (2) : 69-70.

2. BHALERAO (G.). On some representatives of the genus Avitellina from India, J. Helminth, 1936, 14 (3) : 141-162.

3. GRABER (M.). Rapport annuel du Laboratoire de Farcha 1967. T. III : Helminthes et helmunthiases. Bilan d'activité, N'Djaména, Tchad, 1968, 192 p.

4. GRABER (M.). Rapport annuel du Laboratorre de Farcha 1967, T. V : Pathologie de la faune sauvage du Tchad. Premiers résultats d'enquêtes. N'Djaména, Tchad, 1968, $157 \mathrm{p}$.

5. GRABER (M.). Helminthes parasites de certains animaux domestiques et sauvages du Tchad. Bull. epizoot. Dis. Afr., 1969, 17 (4) : 403-428.

6. GRABER (M.), BLANC (Ph.), DELAVENAY (R.). Helminthes des animaux sauvages d'Ethiopie. I. Mammufères. Rev. Elev. Méd. vét. Pays trop. (à paraître).

7. GRABER (M.), BOUCHET (A.), FINELLE (P.), DESROTOUR (J.), MACON (G.). Le parasitisme du zébu dans l'Ouest de la République Centrafricaine. 2. Parasites des bouvillons et des adultes, Rev. Elev. Méd. vét. Pays trop., 1969, 22 (4) : 509-519.

8. GRABER (M), DOUTRE (M.), FINELLE (P.), KERAVEC (J.), DUCROZ (G.), MOKOTAINGAR (P.). Les helminthes de quelques artiodactyles sauvages appartenant aux familles des bovidés et des suidés. Ces mammifères, en République du Tchad et en R. C. A., sont-ils des réservoirs de parasites pour les animaux domestiques vivant à leur contact? Rev. Elev. Méd. vét. Pays trop., 1964, 17 (3) : 377420.

9. HORAK (I. G.). Parasites of domestic and wild animals in South Africa. IX. Helminths of Blesbok. Onderstepoort J. vet. Res., 1978, 45 (2) : 55-58.

10. KEEP (M. E.). Internal and blood parasites of african antelopes. Vet. Rec., 1969, 84 (20) : 514.

11. KHALIL (L. F.), GIBBONS (L. M.).The helminth parasites of the Suni, an african antelope from Kenya. Parasitology, 1975, 71 (2) : XVII.
12. PETISCA (J. L. N.), FAZENDEIRO (M. I.), FERREIRA (M. L.), MATOS (V.). Contribuição para o estudo da estilesiose hepatica em Moçambique. Revta Cienc. vet., 1974, 7 A : 1-27.

13. ROTH (H. H.), DALCHOW (W.). Untersuchungen über Wurmbefall von Antilopen in Rhodesien. $Z$. angew. Zool., 1967, 54 (2) : 203-226.

14. ROUND (M. C.). Check-list of the helminth parasites of african mammals. St. Albans, Commonwealth Bureau of Helminthology, 1968, 252 p. (Technical communication, $n^{\circ} 38$ ).

15. SACHS (R.), HOFMANN (R. R.), SORHEIM (A. O.). Stilesia infestation in the east african antelopes. Vet. Rec., 1969, 84 (9) : 233-234.

16. SACHS (R.), SACHS (C.). A survey of parasitic infestation of wild herbivores in the Serengeti region in northern Tanzania and the lake Rukwa region in southern Tanzania. Bull. epizoot. Dis. Afr., 1968, $16(4): 455-472$

17. SILVA (J. A. C.). Contribuição para o estudo dos helmintos parasitas dos vertebrados de Moçambique. Mem. Junta. Invest. Ultram. 2 Sér., Lisboa, 1971 (61) : $1-479$.

18. SPASSKI (A. A.). Essentials of cestodology. 1. Anoplocephalata tapeworms of domestic and wild animals. Moscou, Acad. Scı. U. R. S. S., 1951 ; Jerusalem, Israel Program for Scientific translations, 1961, $783 \mathrm{p}$.

19. THAL (J.). Les maladies similaires à la peste bovine. Etude et lutte. Rapport. Projet P. N. U. D./C. A. F. 13, Paris, I. E. M. V. T., 1972, 57 p.

20. WOODLAND (W. N. F.). On a new genus of $A v i-$ tellina tapeworms from ruminants in East Africa Parasitology, 1928, 20 (1) : 56-65.

21. WOODLAND (W. N. F.). A new species of Avitellina tapeworm Avitellina sandgroundi from Hippotragus equinus. Ann. trop. Med. Parasit. 1935, 29 (2) : 185-190.

22. YOUNG (E.), VAN DEN HEEVER (L. W.). The african buffalo as a source of food and by-products. J. S. Afr., vet. med. Ass., 1969, 40 (1) : 83-88. 